\title{
Wigner function for free relativistic particles
}

\author{
O. I. Zavialov, A. M. Malokostov
}

\begin{abstract}
A generalization of the Wigner function for the case of a free particle with the
\end{abstract} "relativistic" Hamiltonian $\sqrt{\mathbf{p}^{2}+m^{2}}$ is given.

\section{Introduction}

It is well known [1] that the quantum description of a non-relativistic particle, whose momentum space wave function (at the moment $t=0$ ) is $\psi(\mathbf{p})$, can be handled in the equivalent "classical" form. Namely, one defines the so called Wigner function

$$
\begin{aligned}
W(\mathbf{p}, \mathbf{x}, t)=\frac{1}{(2 \pi)^{3}} \int & d \mathbf{p}_{1} d \mathbf{p}_{2} \psi^{*}\left(\mathbf{p}_{1}\right) \psi\left(\mathbf{p}_{2}\right) \delta\left(\mathbf{p}-\frac{\mathbf{p}_{1}+\mathbf{p}_{2}}{2}\right) \times \\
& \times \exp \left\{i\left(\frac{\mathbf{p}_{1}^{2}}{2 m}-\frac{\mathbf{p}_{2}^{2}}{2 m}\right) t+i\left(\mathbf{p}_{2}-\mathbf{p}_{1}\right) \mathbf{x}\right\} .
\end{aligned}
$$

This quantity can be interpreted (see the survey [3]) as the density distribution over the six-dimensional phase space $\{\mathbf{p}, \mathbf{x}\}$ of the set of classical non-relativistic free, i.e. corresponding to the Hamiltonian (2)

$$
H=\frac{\mathbf{p}^{2}}{2 m}
$$

particles. One can verify that the expectation values $\overline{f(\mathbf{x})}$ and $\overline{\varphi(\mathbf{p})}$ of the functions $f(\mathbf{x})$ and $\varphi(\mathbf{p})$ over this classical ensemble coincide with the corresponding quantum matrix elements:

$$
\begin{aligned}
& \overline{f(\mathbf{x})}=\int d \mathbf{p} d \mathbf{x} W(\mathbf{p}, \mathbf{x}, t) f(\mathbf{x})=\int d \mathbf{x} \widetilde{\psi}^{*}(\mathbf{x}, t) f(\widehat{\mathbf{x}}) \widetilde{\psi}(\mathbf{x}, t) \\
& \overline{\varphi(\mathbf{p})}=\int d \mathbf{p} d \mathbf{x} W(\mathbf{p}, \mathbf{x}, t) \varphi(\mathbf{p})=\int d \mathbf{p} \psi^{*}(\mathbf{p}, t) \varphi(\widehat{\mathbf{p}}) \psi(\mathbf{p}, t)
\end{aligned}
$$

Here

$$
\widetilde{\psi}(\mathbf{x}, t)=\frac{1}{(2 \pi)^{3 / 2}} \int d \mathbf{p} e^{i \mathbf{p x}} \psi(\mathbf{p}, t)
$$

and

$$
\psi(\mathbf{p}, t)=e^{-i \frac{p^{2}}{2 m} t} \psi(\mathbf{p})
$$


are the coordinate and the momentum wave functions at the moment $t$. Moreover, the expectation value of the function $F(\mathbf{p}, \mathbf{x})$ over this classical distribution coincides with the matrix element

$$
\langle\widehat{F}(\widehat{\mathbf{p}}, \widehat{\mathbf{x}})\rangle,
$$

where $\widehat{F}$ is chosen in Weyl quantization.

So, what most impressive in the Wigner's idea is that the quantum evolution and the evolution of the classical ensemble (11) are identical. However, this identity should not be taken too serious: the Wigner function is not necessarily positive.

It is curious that the generalization of relation (11) for the relativistic case, i.e., for the Hamiltonian

$$
H(\mathbf{p})=\omega(\mathbf{p})=\sqrt{\mathbf{p}^{2}+m^{2}}
$$

seems to be still unknown. The aim of the present paper is to give such a generalization.

In order to get more insight in what we are going to construct, let us enumerate the basic properties of the Wigner function (1):

a) It is bilinear with respect to $\psi^{\prime}(\mathbf{p})$.

b) The integral of $W$ over $\mathbf{x}$ is just the probability density in momentum space,

$$
\psi^{\prime *}(\mathbf{p}, t) \psi^{\prime}(\mathbf{p}, t)=\int d \mathbf{x} W(\mathbf{p}, \mathbf{x}, t) .
$$

c) The integral of $W$ over $\mathbf{p}$ is just the probability density in coordinate space,

$$
\widetilde{\psi}^{*}(\mathbf{x}, t) \widetilde{\psi^{\prime}}(\mathbf{x}, t)=\int d \mathbf{p} W(\mathbf{p}, \mathbf{x}, t) .
$$

d) The Wigner function admits the classical evolution law. Namely,

$$
W(\mathbf{p}, \mathbf{x}, t+\tau)=W\left(\mathbf{p}, \mathbf{x}-\frac{\mathbf{p}}{m} \tau, t\right) .
$$

One can easily verify all this but we postpone the corresponding calculations since these results will follow immediately from the relativistic formula in the limit of small momenta.

It is known that the naive quantum mechanical coordinate wave function scheme is incompatible with relativistic principles. Historically this was in fact the main motivation for quantum field theory. For example, one encounters internal difficulties even when constructing the very coordinate operator $\widehat{\mathbf{x}}[2]$. Anyway, suppose that the free particle with the Hamiltonian (5) has the momentum space wave function (the function on the upper hyperboloid) equal to $\psi(\mathbf{p})$. The scalar product between two such functions $\psi_{1}(\mathbf{p})$ and $\psi_{2}(\mathbf{p})$ is

$$
\left\langle\psi_{1}(\mathbf{p}), \psi_{2}(\mathbf{p})\right\rangle=\int d \mu(\mathbf{p}) \psi_{1}^{*}(\mathbf{p}) \psi_{2}(\mathbf{p}),
$$

where $d \mu(\mathbf{p})=\frac{d \mathbf{p}}{\omega(\mathbf{p})}$ is the invariant measure on the mass hyperboloid. It is generally believed that the transformation properties of the momentum space wave function $\psi(\mathbf{p})$ with respect to the Lorentz transformation $\Lambda$ are as follows:

$$
\psi_{\Lambda}(\mathbf{p})=\psi(\boldsymbol{\Lambda} \mathbf{p})
$$


It is natural to go to the momentum space $L_{2}\left(d \mathbf{p}, \mathbb{R}^{3}\right)$ of the wave functions $\psi^{\prime}(\mathbf{p})$ with the Lebesgue measure $d \mathbf{p}$. The corresponding isomorphism is given by the map

$$
\psi^{\prime}(\mathbf{p})=\frac{\psi(\mathbf{p})}{\sqrt{\omega(\mathbf{p})}}
$$

and the general Lorentz transformation law can be of the form

$$
\psi_{\Lambda}^{\prime}(\mathbf{p})=\sqrt{\frac{\omega(\boldsymbol{\Lambda} \mathbf{p})}{\omega(\mathbf{p})}} e^{i \Omega(\boldsymbol{\Lambda} \mathbf{p})-i \Omega(\mathbf{p})} \psi^{\prime}(\boldsymbol{\Lambda} \mathbf{p}) .
$$

Here $\Omega(\mathbf{p})$ is an arbitrary real function.

Thus, the momentum operator $\widehat{\mathbf{p}}$ in this latter space is just the Hermitean multiplication:

$$
\widehat{\mathbf{p}} \psi^{\prime}(\mathbf{p})=\mathbf{p} \psi^{\prime}(\mathbf{p}) .
$$

It follows from the canonical commutational relations that the coordinate operators $\widehat{\mathbf{x}}$ (if any) can be chosen as $\widehat{\mathbf{x}}=i \frac{\partial}{\partial \mathbf{p}}$. Hence, the coordinate space wave function $\widetilde{\psi^{\prime}}(\mathbf{x})$ (if any) is again just the Fourier transform of the momentum space wave function $\psi^{\prime}(\mathbf{p})$ :

$$
\widetilde{\psi^{\prime}}(\mathbf{x}, t)=\frac{1}{(2 \pi)^{3 / 2}} \int d \mathbf{p} e^{i \mathbf{p x}} \psi^{\prime}(\mathbf{p}) e^{-i \omega(\mathbf{p}) t} .
$$

So, let us formulate our problem.

Find the function $W(\mathbf{p}, \mathbf{x}, t)$ which satisfies the following principles, inspired by the non-relativistic treatment.

1) It is bilinear with respect to $\psi^{\prime}(\mathbf{p})$.

2) The integral of $W$ over $\mathbf{x}$ is just the probability density in momentum space,

$$
\psi^{\prime *}(\mathbf{p}, t) \psi^{\prime}(\mathbf{p}, t)=\int d \mathbf{x} W(\mathbf{p}, \mathbf{x}, t) .
$$

3) The integral of $W$ over $\mathbf{p}$ is just the probability density in coordinate space,

$$
\widetilde{\psi}^{*}(\mathbf{x}, t) \widetilde{\psi^{\prime}}(\mathbf{x}, t)=\int d \mathbf{p} W(\mathbf{p}, \mathbf{x}, t) .
$$

4) The Wigner function admits the classical (relativistic) evolution law. Namely,

$$
W(\mathbf{p}, \mathbf{x}, t+\tau)=W\left(\mathbf{p}, \mathbf{x}-\frac{\mathbf{p}}{\omega(\mathbf{p})} \tau, t\right) .
$$

5) In the limit of small momenta it tends to the function $W$ defined by (1).

Practically, the requirements 1)-3) are just the repetition of the corresponding restrictions imposed on the initial Wigner function (1). Note however the different nature of the relativistic wave functions $\psi$ and $\psi^{\prime}$. 
The solution of the problem is given by the formula

$$
\begin{aligned}
W(\mathbf{p}, \mathbf{x}, t)= & \frac{1}{(2 \pi)^{3}} \int d \mathbf{p}_{1} d \mathbf{p}_{2} \psi^{\prime *}\left(\mathbf{p}_{1}\right) \psi^{\prime}\left(\mathbf{p}_{2}\right) \delta\left(\mathbf{p}-\left(\mathbf{p}_{1} \dot{+} \mathbf{p}_{2}\right)\right) \times \\
& \times \exp \left(i\left(\omega\left(\mathbf{p}_{1}\right)-\omega\left(\mathbf{p}_{2}\right)\right) t+i\left(\mathbf{p}_{2}-\mathbf{p}_{1}\right) \mathbf{x}\right) .
\end{aligned}
$$

Here the symbol $\dot{+}$ denotes the special sum on the mass hyperboloid: if we introduce the four-vectors $P_{1}=\left(\omega\left(\mathbf{p}_{1}\right), \mathbf{p}_{1}\right)$ and $P_{2}=\left(\omega\left(\mathbf{p}_{2}\right), \mathbf{p}_{2}\right)$, then, by definition,

$$
P_{1}+P_{2} \equiv m \frac{P_{1}+P_{2}}{\sqrt{\left(P_{1}+P_{2}\right)^{2}}},
$$

and $\mathbf{p}_{1} \dot{+} \mathbf{p}_{2}$ is simply the spatial part of $P_{1} \dot{+} P_{2}$.

In other words,

$$
\mathbf{p}_{1} \dot{+} \mathbf{p}_{2}=m \frac{\mathbf{p}_{1}+\mathbf{p}_{2}}{\sqrt{2\left(m^{2}+\omega\left(\mathbf{p}_{1}\right) \omega\left(\mathbf{p}_{2}\right)-\mathbf{p}_{1} \mathbf{p}_{2}\right)}} .
$$

The proof of the properties 1)-5) is given in the final section of this paper.

Of course, the situation with the physical interpretation of the relativistic function $W$ is not at all better than of the non-relativistic one. Namely, this function can be negative as well. Next, the expectation values over the classical " ensemble" for "mixed" functions (depending simultaneously on $\mathbf{p}$ and $\mathbf{x}$ ) have nothing to do with quantum matrix elements at least in Weyl quantization. Moreover, the general principles of classical mechanics tell us that $W(\mathbf{p}, \mathbf{x}, t)$ should be a scalar with respect to Lorentz transformations. This is by no means consistent with any of the transformation laws of the wave functions (6). It shows that even for free particles the incompatibility of the principles of the conventional quantum mechanics and those of special relativity might be even deeper than it is usually admitted. However, we hope that the function (8) could be still of use in particular reference frames. For example, it can be useful in the theory of "continuous measurements". For us it is interesting in order to analyze the special representation of the

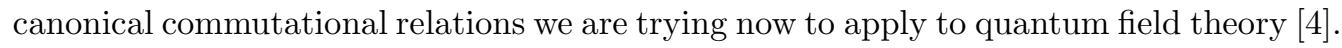

\section{The proof of the relations 1 ) -5 )}

The property 1) is obvious. The property 5) can be also easily seen from (9) in the limit $\omega \rightarrow m$. The property 3) can be verified immediately. Indeed, the integration of $W$ over $\mathbf{p}$ is absorbed by the $\delta$-function. The remainder is just the probability density $\psi^{*}(\mathbf{x}, t) \psi(\mathbf{x}, t)$. The procedure leading to the property 2$)$ is also very transparent. Namely, the integration over $\mathbf{x}$ produces the $\delta$-function:

$$
\frac{1}{(2 \pi)^{3}} \int d \mathbf{x} \exp \left\{i \mathbf{x}\left(\mathbf{p}_{1}-\mathbf{p}_{2}\right)\right\}=\delta\left(\mathbf{p}_{1}-\mathbf{p}_{2}\right)
$$


So, effectively

$$
\mathbf{p}_{2}=\mathbf{p}_{1} .
$$

With this relation, the initial $\delta$-function becomes $\delta\left(\mathbf{p}-\mathbf{p}_{1}\right)$ and the integration over $\mathbf{p}_{1}$ leads to the expected equation 2).

Before turning to 4$)$, let us state the following relation. If $\mathbf{p}=\left(\mathbf{p}_{1} \dot{+} \mathbf{p}_{2}\right)$, then

$$
\omega(\mathbf{p})=\frac{m}{\sqrt{2}} \frac{\omega\left(\mathbf{p}_{1}\right)+\omega\left(\mathbf{p}_{2}\right)}{\sqrt{m^{2}+\omega\left(\mathbf{p}_{1}\right) \omega\left(\mathbf{p}_{2}\right)-\mathbf{p}_{1} \mathbf{p}_{2}}} .
$$

Thus, in order to prove 4), we need only to check that

$$
\omega\left(\mathbf{p}_{2}\right)-\omega\left(\mathbf{p}_{1}\right)=\left(\mathbf{p}_{2}-\mathbf{p}_{1}\right) \frac{\mathbf{p}}{\omega(\mathbf{p})} .
$$

The following chain of transformations makes it obvious.

$$
\omega\left(\mathbf{p}_{2}\right)-\omega\left(\mathbf{p}_{1}\right)=\frac{\omega^{2}\left(\mathbf{p}_{2}\right)-\omega^{2}\left(\mathbf{p}_{1}\right)}{\omega\left(\mathbf{p}_{2}\right)+\omega\left(\mathbf{p}_{1}\right)} .
$$

The left-hand side can be rewritten identically as follows.

$$
\begin{aligned}
\omega\left(\mathbf{p}_{2}\right)-\omega\left(\mathbf{p}_{1}\right)= & \frac{\left(\mathbf{p}_{2}-\mathbf{p}_{1}\right)\left(\mathbf{p}_{2}+\mathbf{p}_{1}\right)}{\sqrt{m^{2}+\omega\left(\mathbf{p}_{1}\right) \omega\left(\mathbf{p}_{2}\right)-\mathbf{p}_{1} \mathbf{p}_{2}}} \times \\
& \times \frac{\sqrt{m^{2}+\omega\left(\mathbf{p}_{1}\right) \omega\left(\mathbf{p}_{2}\right)-\mathbf{p}_{1} \mathbf{p}_{2}}}{\omega\left(\mathbf{p}_{1}\right)+\omega\left(\mathbf{p}_{2}\right)} .
\end{aligned}
$$

After ovious cancellations we arrive just at the desired relation.

For model considerations, it is convenient sometimes to choose the two-dimensional space-time. The momentum space corresponding to the mass-shell $P^{2}=m^{2}$ will be one-dimensional:

$$
P=\{m \operatorname{ch} \Gamma, m \operatorname{sh} \Gamma\} .
$$

In this case the $\dot{+}-\delta$-function can be effectively integrated. One gets for two dimensions:

$$
\begin{aligned}
W(p, x, t)=\frac{2 m^{3}}{\operatorname{ch} \Gamma} \int_{-\infty}^{+\infty} d \beta \operatorname{ch}(\Gamma+\beta) \operatorname{ch}(\Gamma-\beta) \times \\
\quad \times \psi^{\prime *}(m \operatorname{sh}(\Gamma+\beta)) \psi^{\prime}(m \operatorname{sh}(\Gamma-\beta)) \times \\
\quad \times \exp \left\{\frac{i t}{m}[\operatorname{ch}(\Gamma+\beta)-\operatorname{ch}(\Gamma-\beta)]-\frac{i x}{m}[\operatorname{sh}(\Gamma+\beta)-\operatorname{sh}(\Gamma-\beta)]\right\} .
\end{aligned}
$$

Indeed, we pass to analogous variables for one-dimensional momenta $p_{1}$ and $p_{2}$ in (8):

$$
\begin{array}{ll}
\frac{p_{1}}{m}=\operatorname{sh} \gamma_{1}, & \frac{p_{2}}{m}=\operatorname{sh} \gamma_{2}, \\
\frac{d \mathbf{p}_{1}}{m}=\operatorname{ch} \gamma_{1} d \gamma_{1}, & \frac{d \mathbf{p}_{2}}{m}=\operatorname{ch} \gamma_{2} d \gamma_{2} .
\end{array}
$$


Thus, the Wigner function $W$ takes the form:

$$
\begin{aligned}
W=m^{2} & \int d \gamma_{1} d \gamma_{2} \operatorname{ch} \gamma_{1} \operatorname{ch} \gamma_{2} \psi^{\prime *}\left(m \operatorname{sh} \gamma_{1}\right) \psi^{\prime}\left(m \operatorname{sh} \gamma_{2}\right) \times \\
& \times \exp \left\{i \frac{t}{m}\left(\operatorname{ch} \gamma_{1}-\operatorname{ch} \gamma_{2}\right)-i \frac{x}{m}\left(\operatorname{sh} \gamma_{1}-\operatorname{sh} \gamma_{2}\right)\right\} \times \\
& \times m \delta\left(\operatorname{sh} \Gamma-\frac{\operatorname{sh} \gamma_{1}+\operatorname{sh} \gamma_{2}}{\sqrt{2} \sqrt{1+\operatorname{ch} \gamma_{1} \operatorname{ch} \gamma_{2}-\operatorname{sh} \gamma_{1} \operatorname{sh} \gamma_{2}}}\right) .
\end{aligned}
$$

The expression under the sign of the square root in the argument of $\delta$-function can be transformed as follows:

$$
\begin{aligned}
& 1+\operatorname{ch~} \gamma_{1} \operatorname{ch} \gamma_{2}-\operatorname{sh} \gamma_{1} \operatorname{sh} \gamma_{2}=1+\operatorname{ch}\left(\gamma_{1}-\gamma_{2}\right)= \\
& \quad=1+\operatorname{ch}^{2}\left(\frac{\gamma_{1}-\gamma_{2}}{2}\right)+\operatorname{sh}^{2}\left(\frac{\gamma_{1}-\gamma_{2}}{2}\right)=2 \operatorname{ch}^{2}\left(\frac{\gamma_{1}-\gamma_{2}}{2}\right) .
\end{aligned}
$$

So, the total integral (12) takes the form

$$
\begin{aligned}
W & =m^{3} \int d \gamma_{1} d \gamma_{2} \Psi^{*}\left(\gamma_{1}, x, t\right) \Psi\left(\gamma_{2}, x, t\right) \delta\left(\operatorname{sh} \Gamma-\operatorname{sh} \frac{\gamma_{1}+\gamma_{2}}{2}\right) \operatorname{ch} \gamma_{1} \operatorname{ch} \gamma_{2}= \\
& =m^{3} \int d \gamma_{1} d \gamma_{2} \Psi^{*}\left(\gamma_{1}, x, t\right) \Psi\left(\gamma_{2}, x, t\right) \frac{1}{\operatorname{ch} \Gamma} \delta\left(\Gamma-\frac{\gamma_{1}+\gamma_{2}}{2}\right) \operatorname{ch} \gamma_{1} \operatorname{ch} \gamma_{2}
\end{aligned}
$$

where

$$
\Psi(\gamma, x, t)=\psi^{\prime}(m \operatorname{sh} \gamma) \exp \left(-i \frac{t}{m} \operatorname{ch} \gamma+i \frac{x}{m} \operatorname{sh} \gamma\right) .
$$

Let us go to the new variables $\alpha$ and $\beta$ :

$$
\gamma_{1}=\alpha+\beta, \quad \gamma_{2}=\alpha-\beta .
$$

We arrive finally at (11).

The effective "integration" of the $\delta$-function is also possible in the three-dimensional case.

This paper was supported by the Russian Foundation for Basic Research, grant No. 98-01-00162a

\section{References}

[1] Wigner E. P., Phys. Rev., 1932, 40, p. 749.

[2] Newton T. D., Wigner E. P., Rev. Mod. Phys., 1949, 21, p. 400.

[3] V. I. Tatarskii, Uspekhi Fiz. Nauk, 1983, 139, p. 587 (in Russian).

[4] O.I. Zavialov, A. M. Malokostov., TMF, to appear. 